http://jmscr.igmpublication.org/home/ ISSN (e)-2347-176x ISSN (p) 2455-0450 crossref DOI: https://dx.doi.org/10.18535/jmscr/v8i6.70

\title{
Association of dyslipidemia and Diabetic Retinopathy among patients coming to OPD of Katihar Medical College Hospital and its visual outcome: A prospective observational study
}

\author{
Authors \\ Dr Himanshu Kumar ${ }^{1}$, Dr Arjun Kumar Singh ${ }^{2 *}$ \\ ${ }^{1}$ Assistant Professor, Department of Ophthalmology, Katihar Medical College, Katihar, Bihar \\ ${ }^{2}$ Associate Professor, Department of Ophthalmology, Katihar Medical College, Katihar, Bihar \\ *Corresponding Author \\ Dr Arjun Kumar Singh \\ A/39 PC Colony Opposite to Arun Jeitly Park Kankarbagh Patna 800020, Bihar, India
}

\section{Introduction}

Diabetic retinopathy (DR) is one of the major microvascular complications of diabetes. It is one of the most common cause of preventable blindness in diabetic adults. Dyslipidemia, a major systemic disorder, is one of the most important risk factors for cardiovascular disease. Patients with diabetes have an increased risk of suffering from dyslipidemia concurrently.

\section{Aims and Objective of the study}

a) To find out the association between Diabetic Retinopathy (DR) and Lipid Profile.

b) To find out the possible mechanisms involving lipid metabolism and diabetic retinopathy.

c) To find out the effect of lipid-lowering therapies on diabetic retinopathy.

\section{Background}

For traditional lipid markers, evidence is available that total cholesterol and low-density lipoprotein cholesterol are associated with the presence of hard exudates in patients with DR. The study of nontraditional lipid markers is advancing only in recently years. The severity of DR is inversely associated with apolipoprotein A1 (ApoA1), whereas $A p o B$ and the ApoB-to-ApoA1 ratio are positively associated with DR. The role of lipidlowering medication is to work as adjunctive therapy for better control of diabetes-related complications including DR.

\section{Material and Method}

This retrospective study was conducted inthe Ophthalmology Department in Katihar Medical College \& Hospital, Katihar, Bihar from1 January 2018 to 31 December 2019.The study was conducted with the approval of the Institutional Ethics committee with proper consent taken from the patients.

\section{Inclusion Criteria}

Patients with more than 5 years of diagnosed diabetes Type 2 were included.

Duration of diabetes ranged from 5 to 25 years.

Age group 20 to 80 years of age. 


\section{Exclusion Criteria}

a) Patients with history less than 5 years of diagnosed diabetes.

b) Ocular surgery less than 6 months.

c) Those with accelerated hypertension.

d) Active ocular infection.

e) Co-existing ocular disorders such as: -

Uveitis, Opaque or Hazy media, Retinal disorders such as retinal vein or artery occlusions or retinitis pigmentosa, vitreoretinal degenerations, dystrophies, high myopia, glaucoma and cataract were excluded from the study.

\section{Result}

A total of 40 patients were included in the study, there were 26 male and 14 females patients.

Of the 40 patients included in the study, $30(75 \%)$ had DR and10 (25\%) did not have DR. Various grades of CSME were detected in 17 patients $(42.5 \%)$.

Patients were divided into four groups as follows. Group 1 included patients with no DR (the control group).

Group 2 included patients with mild-to-moderate nonproliferative DR with or without haemorrhages.

Group 3 included patients with severe nonproliferative DR with small haemorrhages

Group 4 included patients with proliferative DR with massive HEs.

\section{All patients were subjected to the following}

1. Full history taking: Age of the patient, type of diabetes, duration of diabetes, mode of diabetic control, family history of diabetes mellitus and history of known ocular or medical diseases

\section{Clinical examination included:}

Assessment of visual acuity, Refraction, Tonometry using applanation tonometer, Complete ophthalmological examination including slit-lamp biomicroscopy for anterior segment examination and using 78$D$ lens with or indirect ophthalmoscope with 20-D lens for fundus examinations and best- corrected visual acuity using illuminated Landolt chart

\section{Investigations}

a. Ocular: Fundus Fluorescein Angiography and Optical Coherent Tomography

b. Systemic: Lipid profile measurements using Fasting samples, Fasting and PostPrandial blood sugar and HbAlc.

Serum lipid measurements were carried out using fasting samples.

For the purpose of analysis, dyslipidaemia was defined as follows:

Serum Total cholesterol $>160 \mathrm{mg} / \mathrm{dl}$

Triglyceride levels $>150 \mathrm{mg} / \mathrm{dl}$

Low-density lipoprotein (LDL) levels $>100 \mathrm{mg} / \mathrm{dl}$ High-density lipoprotein (HDL) $<40 \mathrm{mg} / \mathrm{dl}$ for men and less than $50 \mathrm{mg} / \mathrm{dl}$ for women ${ }^{[6]}$. Patients were given antilipidemic therapy according to the type of lipid elevated by medical specialist as follows.

Atorvastatin $20 \mathrm{mg}$ tablet was given daily after dinner for 2 weeks to patients with high total cholesterol or high cholesterol components [LDL and very-low-density lipoprotein (VLDL)].

After 9 months, the investigations were repeated again to see the effect of treatment.

\section{Results}

Between $1^{\text {st }}$ January 2018 to $31^{\text {st }}$ December 2019, 40 diabetic patients underwent this study, of whom $26(65 \%)$ were male and 14 (35\%) were female, and the mean age was 50.5 years (range = 20-80 years). Duration of diabetes ranged from 5 to 25 years. Thirty $(75 \%)$ patients had DR and 10 (25\%) patients did not have DR. various grades of CSME were detected in 12 diabetic patients $(42.5 \%)$.

Prevalence of DR is significantly increased with increasing age $(P<0.05)$. Various stages of CSME were significantly increased with increasing age $(P<0.05)$. Prevalence of DR is significantly increased with increased duration of diabetes. Various stages of CSME were significantly increased with increased duration of diabetes $(P<0.05)$. 
Dyslipidaemia was found in $25(62.5 \%)$ diabetic patients. Dyslipidaemia was found in 21 (70\%) DR patients.

Twelve patients (48\%) out of the 25 dyslipidaemia patients had CSME.

Serum lipid profile, including total cholesterol, LDL, VLDL and triglyceride level were elevated in DR and CSME.The incidence of patients with elevated total cholesterol and triglycerides was $33 \%$ of all DR patients. The incidence of patients with elevated LDL and triglycerides was $23.5 \%$ of all DR patients. The incidence of patients with elevated LDL and VLDL was $20 \%$ of all DR patients. An overall $23.5 \%$ of all DR patients had normal lipid profile.

The percentage of patients with elevated total cholesterol and triglycerides was $30 \%$ of all CSME patients. The percentage of patients with elevated LDL and triglycerides was $23.5 \%$ of all CSME patients. The percentage of patients with elevated LDL and VLDL was $18 \%$ of all CSME patients. An overall 29\% of all CSME patients had normal lipid profile.

Haemorrhages was present in 21 (70\%) DR patients with total cholesterol level above 230 $\mathrm{mg} / \mathrm{dl}$. In addition, hemorrhage was present in $80 \%$ of DR patients with the ratio of total cholesterol level to HDL above 4.5.

Retinal exudate decreased in patients who had exudative DR and took antilipid therapy regularly by about $60 \%$ in all DR patients. Mean visual acuity was $6 / 12$ in group 1 (the control group), $6 / 18$ in group 2, 6/36 in group 3 and below 6/60 in group 4.Mean visual acuity improved one to two lines on Landolt chart to be $6 / 12$ in group 2, 6/18 in group 3 and $6 / 60$ in group 4

\section{Discussion}

It is observed that as the duration of diabetes increases, the chances of having DR and CSME also increase. Various studies have shown an association of dyslipidaemia with macrovascular complications of diabetes (e.g. coronary artery disease), but few have studied the association of serum lipids with microvascular complications such as DR and the available results are conflicting ${ }^{[6],[7]}$.

Early Treatment Diabetic Retinopathy Study (ETDRS) and Wisconsin Epidemiologic Study of Diabetic Retinopathy (WESDR) showed a stronger evidence for the role of serum lipids in exudative maculopathy. In the ETDRS, it was also reported that higher baseline total and LDLcholesterol levels increased the risk for retinal exudation by two-fold. Dornan et al. ${ }^{[6]}$ found that there is an association between total serum cholesterol and DR.

In our study, we found that total serum cholesterol level is directly associated with the severity of retinal HEs in patients with DR and dyslipidaemia. These results were nearly similar to the results gained by Rema et al. ${ }^{[8]}$, who found that individuals with elevated total serum cholesterol, LDL-cholesterol, or triglyceride levels are more likely to have or develop retinal HEs, which can be associated with risk for vision loss, independent of the extent of macular edema. Patients with a total cholesterol/HDL-cholesterol ratio of 4.5 or greater were almost twice as likely to have retinal HEs compared with those with a ratio less than 4.5. Patients with a higher quartile of total cholesterol or LDL-cholesterol levels were 5-6 times more likely to have retinal HEs than those with lowest quartiles. Moreover, patients with elevated total cholesterol $(240 \mathrm{mg} / \mathrm{dl}$ or 6.21 $\mathrm{mmol} / \mathrm{l}$ ) were twice as likely to have retinal HEs at baseline (odds ratio $=2.00 ; 99 \%$ confidence interval $=1.35-2.95$ ).

Similar results were found when comparing the elevated LDL levels $(160 \mathrm{mg} / \mathrm{dl}$ or $1.14 \mathrm{mmol} / \mathrm{l})$ with the lowest level of LDL (130 mg/dl or 3.37 $\mathrm{mmol} / \mathrm{l})$ and the odds ratio was 1.97 (99\% confidence interval $=1.3-2.96$ ) .

Patients with elevated cholesterol and triglyceride levels were $50 \%$ more likely to develop retinal HEs. Elevated serum cholesterol at baseline also increased the risk for visual loss by $50 \%$ compared with lower serum cholesterol levels.

Actions to Control Cardiovascular Risk in Diabetes (ACCORD) ${ }^{[12]}$ is a randomized 
controlled clinical trial with three components, determining the effects of lowering blood glucose, lowering blood pressure, and using fibrates to lower serum triglycerides and raise serum HDLcholesterol levels (on a background of statin treatment) on cardiovascular disease in patients with type 2 diabetes, and a subset of participants with this study will be evaluated with a standardized protocol for comprehensive eye examinations and fundus photography consisting of the seven stereoscopic fields. An important association of DR with total cholesterol and serum triglycerides was showed ${ }^{[8]}$.

High serum triglycerides have also been shown to be associated with the increased risk of the development and progression of retinopathy by Hadjadj et al. ${ }^{[13]}$.

There has been increasing interest in the link between serum lipids and maculopathy in view of the evolving medical treatment. In type 2 diabetic patients, DME showed an association with increased LDL levels ${ }^{[12]}$. Elevated serum cholesterol at baseline also increased the risk for visual loss by $50 \%$ compared with lower serum cholesterol levels ${ }^{[9]}$.

This association was maintained even after adjusting for age, as age by itself is a significant risk factor for hyperlipidaemia. The other significant finding in type 2 diabetes was that DME also showed a strong correlation with high LDL levels in the same study ${ }^{[8]}$.

We also found that the risk for visual acuity loss was associated with both the presence and increasing severity of $\mathrm{HE}$ at baseline, adjusted for the presence and increasing severity of macular oedema.

\section{Conclusion}

DR is one of the most important causes of vision loss worldwide. Serum lipid levels have a significant effect on the severity of retinal HEs. As the density of these HEs increases, they tend to migrate towards the foveal centre where their deposition predisposes to subfoveal fibrosis.
Lowering serum lipids has shown benefit on both proliferative DR and maculopathy.

\section{References}

1. Kulshrestha OP, Nayarand SK, Sharma DP. Role of serum lipids in diabetic retinopathy. Indian J Ophthalmol 1979; 27:116-118.

2. J Idiculla, S Nithyanandam, M Joseph, VKA Mohan, U Vasu, M Sadiq. Serum lipids and diabetic retinopathy: a crosssectional study. Indian J Endocrinol Metab 2012; 16(Suppl 2) :S492-S494.

3. Aiello LP, Avery RL, Arrigg PG, Keyt BA, Jampel HD, Shah ST et al. Vascular endothelial growth factor in ocular fluid of patients with diabetic retinopathy and other retinal disorders. N Engl J Med 1994; $331: 1480-1487$.

4. Sachdev N, Sahni A. Association of systemic risk factors with the severity of retinal hard exudates in a north Indian population with type 2 diabetes. J Postgrad Med 2010; 56 :3-6.

5. Frank RN. Diabetic retinopathy. N Engl J Med 2004; 30 :451-457.

6. Dornan TL, Carter RD, Bron A. Low density lipoprotein cholesterol: an association with the severity of diabetic retinopathy. Diabetologia 1982; 22 :167170.

7. Larsson LI, Alm A, Lithner F, Dahlen G, Bergstrom R. The association of hyperlipidemia with retinopathy in diabetic patients aged 15-50 years in the county of Umea. Acta Ophthalmol Scand 1999; 77 :585-591.

8. Rema M, Srivastava BK, Anitha B, Deepa $\mathrm{R}$, Mohan V. Association of serum lipids with diabetic retinopathy in urban south Indians - The Chennai Urban Rural Epidemiology Study (CURES) Eye Study2. Diab Med 2005; 23 :1029-1036.

9. OM Jew, M Peyman, TC Chen, S Visvaraja. Risk factors for clinically 
significant macular edema in a multiethnics population with type 2 diabetes. Int J Ophthalmol 2012; 5 :499-504.

10. Told DR, Baratsits M, Garhöfe GR, Schmetterer L. Early Treatment Diabetic Retinopathy Study (ETDRS) diabetic retinopathy study design and research group and baseline patient characteristics. 1991; 98:741-756.32. Early Treatment Diabetic Retinopathy Study Report Number 7. ETDRS. Ophthalmology 1991; $98: 741-756$.

11. Klein BEK, Moss SE, Klein R, Surawicz TS. The Wisconsin Epidemiologic Study of Diabetic Retinopathy, XIII: relationship between serum cholesterol to retinopathy and hard exudate. Ophthalmology 1991; $98: 1261-1265$.

12. ACCORD Eye Study Group, Chew EY, Ambrosius WT, Davis MD, Danis RP, Gangaputra S, Greven CM, et al. Actions to Control Cardiovascular Risk in Diabetes (ACCORD) Study Group; Medical Therapies effects on retinopathy Progression in Type 2 diabetes. N Engl J Med 2010; 363 :233-244.

13. Hadjadj S, Duly-Bouhanick B, Bekherraz A, Bridoux F, Gallois Y, Mauco G, et al. Serum triglycerides are predictive factor for the development and the progression of complications in patients with type 1 diabetes. Diabetes Metab 2004; 30 :43-51. 